\title{
Estrés oxidativo: ¿un estado celular defectuoso para la función espermática?
}

\author{
B. José Manuel Mayorga Torres ${ }^{1}$, Mauricio Camargo ${ }^{2}$, Ángela P. Cadavid ${ }^{1}$, Walter D. \\ Cardona Maya ${ }^{1}$. \\ ${ }^{1}$ Grupo Reproducción, Facultad de Medicina, ${ }^{2}$ Grupo Genética, Regeneración y Cáncer -GRC-, Facultad de Ciencias \\ Exactas y Naturales, Universidad de Antioquia. Medellín, Colombia.
}

\section{RESUMEN}

En los organismos vivos, las cantidades de radicales libres y especies reactivas del oxígeno (ROS) son controladas por un complejo sistema de homeostasis, capaz de mantener niveles fisiológicos de ROS necesarios para el funcionamiento y regulación de algunas biomoléculas. Paralelamente, los organismos poseen sistemas bioquímicos de protección contra el estrés oxidativo, que consiste en el desbalance entre la producción de especies químicas altamente reactivas y las defensas antioxidantes de la célula. Dicho estrés contribuye de manera importante a la etiología tanto de la senescencia celular como de algunas enfermedades. En el contexto reproductivo, las células espermáticas pasan por una serie de cambios fisiológicos durante los procesos de maduración, capacitación y fecundados, entre los que se incluyen las modificaciones de las proteínas existentes, reguladas por señales procedentes del entorno espermático, donde las ROS modulan importantes vías bioquímicas, involucradas en procesos fundamentales de la función del espermatozoide y que se pueden alterar en estados de estrés oxidativo. El objetivo de esta revisión de literatura es describir algunos de los procesos que contribuyen al estrés oxidativo y sus implicaciones sobre la funcionalidad espermática.

\section{PALABRAS CLAVE: Espermatozoide, fertilidad, especies reactivas del oxígeno, antioxidantes}

\section{SUMMARY}

Living organisms regulate the load of free radicals and reactive oxygen species (ROS) by a complex homeostatic system, capable of maintaining physiological levels of ROS, necessary for the action and regulation of some biomolecules. In parallel, organisms harbor biochemical protection systems against oxidative stress, consisting of an unbalance state between oxygen reactive chemical species and antioxidant defense production; this kind of biochemical stress has been shown to contribute to cellular senescence and the development of different diseases. In the reproductive field, the spermatic cells undergo a serial of physiological changes during the maturation, capacitation and fertilization process. Such changes include the modification of proteins regulated by signals from the sperm environment, where ROS modulate important biochemical pathways involved in fundamental processes of sperm function, and that could be altered under oxidative stress conditions. The objective of this review is to describe some of the processes that contribute to oxidative stress and its implications on sperm functionality.

KEY WORDS: Sperm, fertility, reactive oxygen species, antioxidants 


\section{INTRODUCCIÓN}

El oxígeno es un compuesto vital para los procesos de la vida aeróbica, que inevitablemente genera las especies reactivas del oxígeno (ROS), agentes de oxidación altamente reactivos, que actúan como intermediarios químicos de vida corta sobre lípidos, aminoácidos, carbohidratos y ácidos nucleicos e inducen mutaciones en la molécula del ADN (1). Cuando la cantidad de ROS sobrepasa el balance entre la producción y la captación, se genera un fenómeno conocido como estrés oxidativo, el cual tiene consecuencias negativas sobre múltiples procesos celulares (2).

Hasta hace pocos años, las ROS eran consideradas elementos tóxicos para los espermatozoides humanos, sin embargo, en la actualidad se acepta que pequeñas cantidades de ROS y sus metabolitos pueden mediar y regular funciones espermáticas como la capacitación espermática, la reacción acrosomal y la interacción intergametos $(3,4)$. No obstante, las ROS deben inactivarse continuamente para mantener un balance celular funcional (5), y es durante este proceso, que los sistemas de detoxificación tienen relevancia.

Las mitocondrias son consideradas una de las principales fuentes celulares de ROS, regulando los diferentes procesos fisiológicos de óxido/reducción (redox) dentro de los que se incluyen: i) la transducción de señales intracelulares $(6,7)$, ii) la respuesta al estrés oxidativo (8-10), iii) el desarrollo embrionario (11), iv) la proliferación y adhesión celular $(12,13)$, v) la expresión genética, y vi) la apoptosis (14). En consecuencia, las mitocondrias, los leucocitos presentes en el eyaculado y los espermatozoides morfológicamente anormales, pueden generar un cambio sobre el estado basal de los agentes oxidantes e inducir cambios sobre la actividad espermática. El objetivo de esta revisión de literatura es describir algunos de los procesos que contribuyen al estrés oxidativo y sus implicaciones sobre la funcionalidad espermática.

\section{MATERIALES Y MÉTODOS}

Se realizó una revisión de la literatura científica relacionada con el estrés oxidativo y la función espermática en la base de datos PUBMED. Se seleccionaron los artículos de revisión, de opinión, reportes de caso y artículos originales, con el fin de obtener la mayor y más actualizada información del tema de interés.

Fuentes de las especies reactivas del oxígeno. Las ROS son generadas como productos secundarios de procesos metabólicos entre los que se encuentran la producción de energía, la detoxificación de compuestos nocivos y la defensa frente a agentes patógenos. Generalmente son producidas en las células por oxidasas y peroxidasas como la xantina oxidasa o como un producto alternativo de enzimas como la óxido-nítrico sintasa o el citocromo p450 (15). En el eyaculado, las principales fuentes de ROS son los leucocitos y las células espermáticas anormales.

Los leucocitos son un componente esencial del sistema inmune innato, son reclutados hacia los sitios de infección por factores quimiotácticos y son capaces de eliminar los microorganismos a través de la fagocitosis. El reconocimiento de un agente patógeno promueve la iniciación de diversas vías de transducción de señales intracelulares, incluyendo el complejo NADPH-oxidasa (16) lo cual induce la producción de varios tipos de ROS como superóxido, peróxido de hidrógeno y ácido hipocloroso (17). Después de la fagocitosis, los microorganismos son eliminados por la exposición a altas concentraciones de ROS y otros productos microbicidas contenidos en los gránulos celulares. Los leucocitos pueden estar presentes en la muestra seminal y son considerados como normales en cantidades inferiores a 1 × 106/mL (18), un aumento podría ser un indicador de un proceso infeccioso.

Los leucocitos y en especial los activados, son capaces de producir por lo menos 100 veces más cantidad de ROS que las células germinales, generando un efecto negativo sobre las espermatogonias (19). Sin embargo, aún no es claro si la presencia de leucocitos en una muestra seminal es necesariamente negativa, debido a que un cierto número de éstos podría llegar atener un efecto beneficioso sobre la concentración, la movilidad y la reacción acrosomal espermática (20). Inclusive, los leucocitos podrían estar promoviendo la eliminación de los espermatozoides defectuosos e influyendo en la estimulación de las funciones espermáticas a través de la liberación de ROS. Así, la presencia de estas células puede tener una función dual en el eyaculado, aún más cuando el impacto negativo de las ROS producidas por los leucocitos depende de los sistemas antioxidantes que regulan la capacidad detoxificante.

Otra de las fuentes de ROS en el semen son los espermatozoides, cuyas mitocondrias son su principal fuente energética y contribuyentes importantes de ROS intracelulares (21). Se estima que el oocito humano contiene más de 100.000 mitocondrias, mientras que los espermatozoides poseen un número menor debido a su reducción durante la espermiogénesis. Durante los cambios morfológicos asociados con la diferenciación a espermátide después de la finalización de la meiosis, y en particular durante la formación del axonema, todas las mitocondrias se agregan hacia el núcleo haploide formando una serie de capas concéntricas, en donde las mitocondrias se encuentran a lo largo de esta estructura, favoreciendo la transferencia óptima de ATP a los motores de dineína del axonema, para dirigir el movimiento flagelar (22). 
Las implicaciones sobre cómo las mitocondrias producen ROS, son de vital importancia para entender una serie de temas biomédicos como la diabetes, el envejecimiento, la enfermedad de Alzheimer, la insuficiencia cardíaca, entre otras condiciones médicas asociadas (23-25). Los niveles basales de ROS y el papel de las mitocondrias en el mantenimiento de éstos aún se desconocen en las células germinales masculinas. Las mutaciones mitocondriales tienen un efecto directo sobre su metabolismo, llegando a producir crisis energética en los espermatozoides, lo que aumenta su deterioro funcional (26). Adicionalmente, existe una relación entre una baja calidad espermática y la generación de ROS, debida a defectos sobre los mecanismos de extrusión citoplasmática. Se ha observado una correlación positiva entre la retención residual de citoplasma por los espermatozoides y la generación de ROS intracelular y se especula que un aumento de estos agentes está mediado por la acción de la enzima citosólica glucosa 6-fosfatodeshidrogenasa $(27,28)$. De otro lado, el incremento de espermatozoides inmaduros con citoplasma residual observado en parejas infértiles, sugiere que la calidad de la espermatogénesis es menor a la de parejas fértiles (29-31). Además, existen otras posibilidades sobre la generación de ROS intracelular en el espermatozoide: i) la fuga de electrones de la cadena de transporte mitocondrial, ii) la NADPH oxidasa como una posible fuente de ROS y, iii) la generación de ROS por medio del oxido nítrico (ON) en la regiones post-acrosomales y ecuatoriales (32).

Varias aproximaciones metodológicas permiten la cuantificación de ROS espermáticas: quimioluminiscencia, espectroscopía y citometría de flujo $(18,33)$. El ensayo de quimioluminiscencia evalúa el estado redox de los espermatozoides con alta sensibilidad, sin embargo, la fuente de éstas en el semen no está claramente identificada, convirtiéndose en un factor de confusión en este análisis.

La espectroscopía de resonancia de espín electrónico es otra técnica que consiste en la evaluación de la reactividad por medio de la detección de las especies químicas que tienen electrones desapareados. Debido a la poca cantidad de muestra y la corta vida de las ROS, su cuantificación mediante citometría de flujo (33), emplea moléculas señal que permiten determinar la cantidad de estos oxidantes intracelulares generadas por las células presentes en el eyaculado, tales como células espermatogénicas maduras e inmaduras, leucocitos, bacterias y algunas células epiteliales.

Función espermática: influencia de las especies reactivas del oxígeno. En general, la incubación artificial de los espermatozoides con bajas concentraciones de ROS estimula la hiperactivación y la capacidad del espermatozoide para realizar la reacción acrosomal, lo que promueve la fusión al oocito $(34,35)$. La primera evidencia experimental de la participación de ROS en la fisiología del espermatozoide humano proviene de la observación de espermatozoides expuestos a oxígeno, los cuales mostraron características de espermatozoides capacitados (36).

Durante las primeras etapas iniciales de la capacitación, las concentraciones de calcio intracelular y el AMP comienzan a elevarse, se inicia la generación de ROS y los espermatozoides adquieren una movilidad vigorosa conocida como hiperactivación. Durante este proceso se genera un aumento global de la fosforilación de la tirosina, como consecuencia del estado redox (37) y del aumento en la generación de AMPc celular (38), proceso indispensable, debido a que si no se genera la fosforilación adecuada, la capacitación espermática se vería afectada (39).

La reducción de peróxido de hidrogeno disminuye considerablemente el proceso de hiperactivación y capacitación espermática $(4,37,40)$, restringiendo incluso la respuesta a otros inductores de la actividad espermática como ionóforo de calcio A23187, liso-fosfatidilcolina, ZP3 y progesterona $(37,41)$, sin llegar a afectar la viabilidad y la movilidad espermática.

Dado que no existen datos sobre las concentraciones fisiológicas de ROS en las que se están desarrollando estos procesos, los tratamientos in vitro intentan recrear algunas condiciones celulares específicas. Sin embargo, la observación de que las células del cúmulo de oocitos bovinos mejora la tasa de fecundación de manera dependiente de oxígeno indica que el oocito estimula a los espermatozoides mediante una serie de moléculas mensajeras para completar el proceso de capacitación, promoviendo la habilidad para fecundar (42).

En 2010, reportaron que gran parte del daño del ADN en los espermatozoides es generado por el estrés oxidativo (43); generalmente más del $96 \%$ de los espermatozoides de una muestra seminal poseen características anormales (18), que al parecer serían un remanente de células morfológicamente anormales que no alcanzaron a completar el proceso. Elevadas concentraciones de ROS median las rupturas en el ADN, fenómeno comúnmente observado en los espermatozoides de hombres infértiles $(34,44,45)$. De hecho, en estudios en los cuales los espermatozoides fueron expuestos a altas concentraciones de ROS producidas artificialmente, se observó un incremento significativo en el daño del ADN, una disminución de la movilidad progresiva y la inducción de procesos apoptóticos $(46,47)$.

Se ha reportado que los daños en el ADN de los espermatozoides pueden ocurrir en el testículo, el epidídimo o después de la eyaculación (48) y las causas pueden estar relacionadas con fallas en el empaquetamiento, la madurez nuclear, la 
fragmentación de la cromatina, aneuploidías o por defectos en la integridad del ADN (49). Diferentes autores proponen la importancia de la compactación del ADN en la protección de la información genética masculina frente a diferentes factores tanto internos como externos $(33,49-53)$.

La membrana plasmática de los espermatozoides posee una composición específica con un contenido alto de ácidos grasos polinsaturados, plasmalógenos y esfingomielinas haciéndola un blanco fácil de los agentes oxidantes. Aunque esta estructura facilita su flexibilidad y funcionalidad, constituye el principal sustrato para la lipoperoxidación, lo que daría lugar a severos desórdenes funcionales afectando mecanismos tan importantes como la adhesión y la fusión con el oocito (1). La oxidación de lípidos causada por condiciones de estrés oxidativo durante el transporte de los espermatozoides a través del epidídimo, afecta a la membrana plasmática además de procesos como la reacción del acrosoma sin generar ningún efecto aparente sobre la movilidad (54).

Como es bien conocido, la apoptosis es un principio biológico de muerte celular programada presente en todos los tipos celulares (55). Este proceso altamente regulado, asociado a una cascada de eventos secuenciales, es inducido por dos posibles vías que llevan a muerte celular: la vía extrínseca es dirigida por ligandos de muerte extracelulares como CD95L/FasL, y la vía intrínseca es inducida por alteraciones mitocondriales, una alteración de la homeostasis celular como consecuencia del estrés inducido por una amplia variedad de factores que incluyen ataque por ROS, el desprendimiento de la matriz extracelular, el calor, la hipoxia, la irradiación con rayos gamma, daños en el ADN, o simplemente la falta de factores de supervivencia.

Existen dos grandes propuestas sobre el mecanismo de apoptosis en espermatozoides maduros: i) La eliminación las células germinales alteradas y ii) la regulación de la senescencia de una célula espermática del aparato reproductor masculino y femenino después de la pérdida de la viabilidad. Se sugiere que la contribución de ROS mitocondrial puede ser un mecanismo apoptótico activado, y por tal razón la mayor parte del daño observado sobre el material genético en los espermatozoides humanos tiene un origen oxidativo (56).

Daños en el ADN espermático son una sólida señal de baja calidad seminal $(33,48-51)$, no obstante, el desarrollo de tecnologías en la reproducción asistida, han permitido el desarrollo de embriones in vitro, a partir de espermatozoides que habrían sido rechazados in vivo, lo cual puede tener serías implicaciones a largo plazo en la salud y el bienestar de los niños concebidos mediante técnicas de reproducción asistida, siendo un objetivo claro, la selección de gametos con las mejores características.
Capacidad antioxidante en el eyaculado. La generación intracelular de ROS per se, es una consecuencia inevitable y fisiológicamente importante; para contrarrestarlo, las mitocondrias y la célula en general, poseen numerosos sistemas de defensa, por lo cual la verdadera fuente de estrés oxidativo es el producto del desequilibrio espacio-temporal de la producción de ROS y los sistemas de desintoxicación (57).

Si bien todos los organismos aeróbicos están continuamente expuestos a ROS, un sistema antioxidante es necesario para secuestrar estos agentes oxidativos y mantener condiciones homeostáticas, sistemas que posiblemente han evolucionado conjuntamente para mantener las concentraciones basales de ROS y antioxidantes evadiendo los efectos patológicos generados por el estrés oxidativo. Estudios en modelos animales en los que se induce la reducción de la defensa antioxidante, confirman las importantes funciones de enzimas como la manganeso superóxidodismutasa, la glutatión peroxidasa y la superóxidodismutasa (3,58-60).

La cantidad de enzimas antioxidantes en el espermatozoide es limitada debido a la reducción del citoplasma celular durante la gametogénesis, lo que hace a los espermatozoides susceptibles al daño oxidativo, y paradójicamente, la membrana espermática en mamíferos presenta una composición lipídica altamente susceptible al ataque oxidativo.

El plasma seminal está formado por la contribución de fluidos que provienen del epidídimo y las glándulas sexuales accesorias: cerca del 5\% deriva de las glándulas de Cowper y las glándulas de Littré, siendo la primera parte del eyaculado; la segunda porción es otorgada por la próstata (15$30 \%$ ), mientras que la última porción es aportada por el epidídimo y las vesículas seminales, en los que sus componentes no están completamente mezclados durante la eyaculación (61). El eyaculado contiene muchas proteínas de importancia para la fecundación, por lo que algunos estudios se han encargado de comparar su composición entre individuos fértiles e infértiles con el objetivo de caracterizarlo $(62,63)$ y asociarlo con la movilidad y la calidad del ADN espermático (64). En el plasma seminal se encuentra una cantidad de antioxidantes, principalmente extracelulares, tanto enzimáticos como no enzimáticos (1), entre los que se incluyen la catalasa, la superóxido dismutasa y el glutatión.

La manipulación, criopreservación y descongelación o centrifugación de las muestras seminales durante los procedimientos de rutina en los laboratorios de reproducción asistida, promueven la producción de ROS y la eliminación de gran parte de los antioxidantes extracelulares, lo cual genera efectos no deseados sobre las células germinales $(65,66)$. 
Beneficios y riesgos de la terapia antioxidante en el manejo de la infertilidad. Debido a los efectos nocivos generados por las ROS, el uso de antioxidantes aparece como una herramienta para proteger a las células del daño oxidativo. La terapia antioxidante ha obtenidos buenos resultados en diferentes investigaciones (67-69); no obstante, son pocos los estudios de intervención que muestren un efecto protector sobre las células espermáticas (51).

Los antioxidantes naturales están adquiriendo gran importancia para la protección de las células frente al daño oxidativo. La melatonina, reduce los niveles de calcio y de la caspasa 9, bloqueando el proceso apoptótico, lo que indica que el mecanismo de acción de la melatonina genera su efecto sobre las mitocondrias espermáticas (70). Del mismo modo, el mio-inositol, la forma más importante del inositol en la naturaleza y un componente de la vitamina $B$, ha demostrado mejorar la función mitocondrial de los pacientes oligoastenoteratozooespérmicos, lo que sugiere que esta molécula puede ser usada para el tratamiento de la infertilidad masculina (71).

Dado que el estrés oxidativo no es un fenómeno localizado y es causado por diferentes agentes oxidantes, es razonable suponer que el tratamiento con una combinación de antioxidantes de biodispobilidad oral y dirigidos hacia el tracto reproductivo masculino, podrían ser una mejor protección frente esta condición (50). No obstante, algunas "recetas" pueden combinar un gran número de antioxidantes con un mecanismo de acción agresivo, aumentando la posibilidad de generar un fenómeno denominado "estrés reductivo" (72), debido a la disminución de las concentración fisiológica de ROS, lo cual es crítico para la función normal de la espermatozoide. Es importante señalar que cerca de un $40 \%$ de los hombres que consultan a una clínica de fertilidad, son fértiles y carentes de daño oxidativo (73) y por tanto no requieren tratamiento antioxidante dado que de hecho, este tipo de terapias podría reducir su potencial de fertilidad, por lo que el consumo de antioxidantes para mejorar la calidad espermática debe tener una base diagnóstica.

\section{CONCLUSIONES}

El estrés oxidativo juega un papel importante en la etiología de la infertilidad masculina (33), aunque los orígenes de las ROS responsables de esta condición han sido objeto de una considerable incertidumbre y de cierta controversia. Si bien existen otras fuentes celulares, la contribución por parte de la mitocondria espermática ha sido estudiada como un factor regulador de la actividad y funcionalidad espermática, dadas las características únicas de estas células al estar en contacto con el ambiente externo. Una área de gran relevancia sería la elucidación del factor asociado a la susceptibilidad frente al daño oxidativo, ya que se ha observado una variación inter individuo y por tanto cobrará un valor predictivo para el desarrollo posterior de las enfermedades asociadas. Adicionalmente, se ha observado un efecto beneficioso en los espermatozoides humanos con la administración de suplementos in vitro de diferentes compuestos antioxidantes naturales 0 sintetizados químicamente. Sin embargo, se ha demostrado que su efecto podría disminuir las concentraciones de las ROS a valores inadecuados y llegar a comportarse como un agente prooxidante. Por tal motivo, el mecanismo de acción, las combinaciones y las dosis de los tratamientos antioxidantes deben ser estudiadas en detalle para evitar efectos secundarios no deseados.

Agradecimientos: Este trabajo fue financiado por la Estrategia de Sostenibilidad del Grupo GRC y Grupo Reproducción de la Universidad de Antioquia.

\section{REFERENCIAS}

1. Sanocka D, Kurpisz M. Reactive oxygen species and sperm cells. Reprod Biol Endocrinol 2004;2(12):12.

2. Agarwal A, Saleh RA. Role of oxidants in male infertility: rationale, significance, and treatment. Urol Clinics North Am 2002;29(4):817-27.

3. Aitken RJ, Clarkson JS, Fishel S. Generation of reactive oxygen species, lipid peroxidation, and human sperm function. Biol Reprod 1989;41(1):183-97.

4. Griveau JF, Le Lannou D. Reactive oxygen species and human spermatozoa: physiology and pathology. Int J Androl 1997;20(2):61-9.

5. Sies H. Strategies of antioxidant defense. EJB Reviews 1993: Springer; 1994. p. 101-7.

6. Nakamura $\mathrm{H}$, Nakamura K, Yodoi J. Redox regulation of cellular activation. Annual Review Immunology 1997;15(1):351-69.

7. Suzuki YJ, Forman HJ, Sevanian A. Oxidants as stimulators of signal transduction. Free Radical Bio Med 1997;22(1-2):269-85.

8. Aslund F, Beckwith J. Bridge over troubled waters: sensing stress by disulfide bond formation. Cell 1999;96(6):751-3.

9. Jakob U, Muse W, Eser M, Bardwell JC. Chaperone activity with a redox switch. Cell 1999;96(3):341-52.

10. Zheng M, Aslund F, Storz G. Activation of the OxyR transcription factor by reversible disulfide bond formation. Science 1998;279(5357):1718-21.

11. Pierce GB, Parchment RE, Lewellyn AL. Hydrogen peroxide as a mediator of programmed cell death in the blastocyst. Differentiation 1991;46(3):181-6.

12. Murrell GA, Francis MJ, Bromley L. Modulation of fibroblast proliferation by oxygen free radicals. Biochem J 1990;265(3):659-65.

13. Novogrodsky A, Ravid A, Rubin AL, Stenzel KH. Hydroxyl radical scavengers inhibit lymphocyte mitogenesis. Proc Nati Acad Sci USA. 1982;79(4):1171-4. 
14. Lennon SV, Martin SJ, Cotter TG. Dose-dependent induction of apoptosis in human tumour cell lines by widely diverging stimuli. Cell Prolif 1991;24(2):203-14.

15. Halliwell $B$. Free radicals and other reactive species in disease. eLS 2005.

16. Nathan C. Neutrophils and immunity: challenges and opportunities. Nat Rev Immunol 2006;6(3):173-82.

17. Nauseef WM. How human neutrophils kill and degrade microbes: an integrated view. Immunol Rev 2007;219(1):88-102.

18. Organization WH. WHO laboratory manual for the examination and processing of human semen: World Health Organization; 2010.

19. de Lamirande E, Gagnon C. Capacitation-associated production of superoxide anion by human spermatozoa. Free Radic Biol Med 1995;18(3):487-95.

20. Chakroun-Feki N, Therond $P$, Couturier M, Eustache F, Limea G, Legrand A, et al. Human sperm quality and lipid content after migration into normal ovulatory human cervical mucus containing low numbers of leukocytes. Asian J Androl 2009;11(3):308-16.

21. Koppers AJ, De luliis GN, Finnie JM, McLaughlin $E A$, Aitken RJ. Significance of mitochondrial reactive oxygen species in the generation of oxidative stress in spermatozoa. J Clin Endocrinol Metab 2008;93(8):3199-207.

22. Chemes HE, Rawe VY. The making of abnormal spermatozoa: cellular and molecular mechanisms underlying pathological spermiogenesis. Cell Tissue Res 2010;341(3):349-57.

23. Matsushima S, Ide T, Yamato M, Matsusaka $H$, Hattori $\mathrm{F}$, Ikeuchi $\mathrm{M}$, et al. Overexpression of mitochondrial peroxiredoxin-3 prevents left ventricular remodeling and failure after myocardial infarction in mice. Circulation 2006;113(14):1779-86.

24. Newsholme P, Haber EP, Hirabara SM, Rebelato EL, Procopio J, Morgan D, et al. Diabetes associated cell stress and dysfunction: role of mitochondrial and nonmitochondrial ROS production and activity. J Physiol 2007;583(Pt 1):9-24.

25. Moreira PI, Cardoso SM, Santos MS, Oliveira CR. The key role of mitochondria in Alzheimer's disease. Journal of Alzheimer's disease : JAD. 2006;9(2):10110.

26. Aitken RJ, Clarkson JS. Cellular basis of defective sperm function and its association with the genesis of reactive oxygen species by human spermatozoa. $J$ Reprod Fertil 1987;81(2):459-69.

27. Aitken RJ. Molecular mechanisms regulating human sperm function. Molec Human Reprod 1997;3(3):16973.

28. Agarwal A, Nallella KP, Allamaneni SS, Said TM. Role of antioxidants in treatment of male infertility: an overview of the literature. Reproductive Biomedicine online 2004;8(6):616-27.

29. Gil-Guzman E, Ollero M, Lopez MC, Sharma RK, Alvarez JG, Thomas AJ, et al. Differential production of reactive oxygen species by subsets of human spermatozoa at different stages of maturation. Hum Reprod 2001;16(9):1922-30.

30. Ollero M, Gil-Guzman E, Lopez MC, Sharma RK, Agarwal A, Larson $\mathrm{K}$, et al. Characterization of subsets of human spermatozoa at different stages of maturation: implications in the diagnosis and treatment of male infertility. Hum Reprod 2001;16(9):1912-21.
31. Aziz N, Saleh RA, Sharma RK, Lewis-Jones I, Esfandiari N, Thomas AJ, et al. Novel association between sperm reactive oxygen species production, sperm morphological defects, and the sperm deformity index. Fertil Steril 2004;81(2):349-54.

32. Vernet P, Fulton N, Wallace C, Aitken RJ. Analysis of reactive oxygen species generating systems in rat epididymal spermatozoa. Biol Reprod 2001;65(4):110213.

33. Mayorga-Torres BJ, Cardona-Maya W, Cadavid A, Camargo M. Evaluacion de los parametros funcionales espermaticos en individuos infertiles normozooespermicos. Actas Urologicas Espanolas. 2013;37(4):221-7.

34. Aitken RJ, Irvine DS, Wu FC. Prospective Analysis of Sperm-Oocyte Fusion and Reactive Oxygen Species Generation as Criteria for the Diagnosis of Infertility. Am J Obstet Gynecol 1991;164(2):542-51.

35. Kodama H, Kuribayashi Y, Gagnon C. Effect of sperm lipid peroxidation on fertilization. Journal of andrology. 1996;17(2):151-7.

36. de Lamirande E, Gagnon C. A positive role for the superoxide anion in triggering hyperactivation and capacitation of human spermatozoa. Int $\mathrm{J}$ Androl 1993;16(1):21-5.

37. Aitken RJ, Paterson M, Fisher H, Buckingham DW, van Duin M. Redox regulation of tyrosine phosphorylation in human spermatozoa and its role in the control of human sperm function. J Cell Sci 1995;108 ( Pt 5)(5):2017-25.

38. Visconti PE, Moore GD, Bailey JL, Leclerc P, Connors SA, Pan D, et al. Capacitation of mouse spermatozoa. II. Protein tyrosine phosphorylation and capacitation are regulated by a cAMP-dependent pathway. Development 1995;121(4):1139-50.

39. Aitken RJ, Buckingham DW, Harkiss D, Paterson $M$, Fisher $\mathrm{H}$, Irvine DS. The extragenomic action of progesterone on human spermatozoa is influenced by redox regulated changes in tyrosine phosphorylation during capacitation. Mol Cell Endocrinol 1996;117(1):83-93.

40. Griveau JF, Renard P, Le Lannou D. An in vitro promoting role for hydrogen peroxide in human sperm capacitation. Int J Androl 1994;17(6):300-7.

41. Bize I, Santander G, Cabello P, Driscoll D, Sharpe C. Hydrogen peroxide is involved in hamster sperm capacitation in vitro. Biol Reprod 1991;44(3):398-403.

42. Blondin P, Coenen K, Sirard MA. The impact of reactive oxygen species on bovine sperm fertilizing ability and oocyte maturation. J Androl 1997;18(4):454-60.

43. Aitken RJ, De luliis GN. On the possible origins of DNA damage in human spermatozoa. Mol Human Reprod 2010;16(1):3-13.

44. Fraga CG, Motchnik PA, Wyrobek AJ, Rempel DM, Ames BN. Smoking and low antioxidant levels increase oxidative damage to sperm DNA. Mutation Res 1996;351(2):199-203.

45. Sun JG, Jurisicova A, Casper RF. Detection of deoxyribonucleic acid fragmentation in human sperm: correlation with fertilization in vitro. Biol Reprod 1997;56(3):602-7.

46. Twigg J, Irvine DS, Houston P, Fulton N, Michael L, Aitken RJ. latrogenic DNA damage induced in human spermatozoa during sperm preparation: protective significance of seminal plasma. Mol Human Reprod 1998;4(5):439-45. 
47. Agarwal A, Said TM. Role of sperm chromatin abnormalities and DNA damage in male infertility. Human Reprod update 2003;9(4):331-45

48. Lewis SEM, Aitken RJ. DNA damage to spermatozoa has impacts on fertilization and pregnancy. Cell Tissue Res 2005;322(1):33-41.

49. Aziz N, Agarwal A. Evaluation of Sperm Damage: Beyond the Who Criteria. Infertil Assisted Reprod 2008:161-77.

50. Lewis SE, John Aitken R, Conner SJ, luliis GD, Evenson DP, Henkel R, et al. The impact of sperm DNA damage in assisted conception and beyond: recent advances in diagnosis and treatment. Reprod Biomed online 2013;27(4):325-37.

51. Gil-Villa AM, Cardona-Maya W, Agarwal A, Sharma $R$, Cadavid Á. Role of male factor in early recurrent embryo loss: do antioxidants have any effect? Fertil Steril 2009;92(2):565-71.

52. Gil-Villa AM, Cardona-Maya W, Agarwal A, Sharma R, Cadavid A. Assessment of sperm factors possibly involved in early recurrent pregnancy loss. Fertil Steril 2010;94(4):1465-72.

53. Rodriguez E, Gil-Villa AM, Aguirre-Acevedo DC, Cardona-Maya W, Cadavid AP. Evaluación de parámetros seminales no convencionales en individuos cuyas parejas presentan muerte embrionaria temprana recurrente: en busca de un valor de referencia. Biomedica 2011;31(1):100-7.

54. Oehninger S, Blackmore P, Mahony M, Hodgen G. Effects of hydrogen peroxide on human spermatozoa. J Assisted Reprod Genetics 1995;12(1):41-7.

55. Kerr JF, Wyllie AH, Currie AR. Apoptosis: a basic biological phenomenon with wide-ranging implications in tissue kinetics. Br J Cancer. 1972;26(4):239-57.

56. De luliis GN, Thomson LK, Mitchell LA, Finnie JM, Koppers AJ, Hedges A, et al. DNA damage in human spermatozoa is highly correlated with the efficiency of chromatin remodeling and the formation of 8-hydroxy२П-deoxyguanosine, a marker of oxidative stress. Biol Reprod 2009;81(3):517-24.

57. Andreyev AY, Kushnareva YE, Starkov AA. Mitochondrial metabolism of reactive oxygen species. Biochem Biokhimiia 2005;70(2):200-14

58. Zeng L, Ji X, Zhang Y, Miao X, Zou C, Lang $\mathrm{H}$, et al. MnSOD expression inhibited by electromagnetic pulse radiation in the rat testis. Electromagnetic Biol Med 2011;30(4):205-18.

59. Schneider M, Forster H, Boersma A, Seiler A, Wehnes $\mathrm{H}$, Sinowatz $\mathrm{F}$, et al. Mitochondrial glutathione peroxidase 4 disruption causes male infertility. FASEB journal: official publication of the Federation of American Societies for Experimental Biology 2009;23(9):323342.

60. Otasevic V, Korac A, Vucetic M, Macanovic B, Garalejic $\mathrm{E}$, Ivanovic-Burmazovic $\mathrm{I}$, et al. Is manganese (II) pentaazamacrocyclic superoxide dismutase mimic beneficial for human sperm mitochondria function and motility? Antioxi Redox Signal 2013;18(2):170-8.
61. Owen DH, Katz DF. A review of the physical and chemical properties of human semen and the formulation of a semen simulant. J Androl 2005;26(4):459-69.

62. Yamakawa K, Yoshida K, Nishikawa H, Kato T, Iwamoto T. Comparative analysis of interindividual variations in the seminal plasma proteome of fertile men with identification of potential markers for azoospermia in infertile patients. J Andrology 2007;28(6):85865.

63. Cadavid J AP, Alvarez A, Markert UR, Cardona-Maya W. Differential protein expression in seminal plasma from fertile and infertile males. J Human Reprod Sci 2014;7(3):206-11.

64. Intasqui P, Camargo M, Del Giudice PT, Spaine DM, Carvalho VM, Cardozo $\mathrm{KH}$, et al. Unraveling the sperm proteome and post-genomic pathways associated with sperm nuclear DNA fragmentation. J Assisted Reprod Genetics 2013;30(9):1187-202.

65. Agarwal A, Ikemoto I, Loughlin KR. Relationship of sperm parameters with levels of reactive oxygen species in semen specimens. J Urol 1994;152(1):107-10.

66. Kumar R, Jagan Mohanarao G, Arvind, Atreja SK. Freeze-thaw induced genotoxicity in buffalo (Bubalus bubalis) spermatozoa in relation to total antioxidant status. Mol Biol Rep 2011;38(3):1499-506.

67. Keskes-Ammar L, Feki-Chakroun N, Rebai T, Sahnoun Z, Ghozzi H, Hammami S, et al. Sperm oxidative stress and the effect of an oral vitamin $E$ and selenium supplement on semen quality in infertile men. Arch Androl 2003;49(2):83-94.

68. Gharagozloo P, Aitken RJ. The role of sperm oxidative stress in male infertility and the significance of oral antioxidant therapy. Hum Reprod 2011;26(7):162840.

69. Safarinejad MR, Safarinejad S. Efficacy of selenium and/or N-acetyl-cysteine for improving semen parameters in infertile men: a double-blind, placebo controlled, randomized study. J Urol 2009;181(2):741-51.

70. Espino J, Bejarano I, Ortiz A, Lozano GM, Garcia JF, Pariente JA, et al. Melatonin as a potential tool against oxidative damage and apoptosis in ejaculated human spermatozoa. Fertil Steril 2010;94(5):1915-7.

71. Condorelli RA, La Vignera S, Di Bari F, Unfer V, Calogero AE. Effects of myoinositol on sperm mitochondrial function in-vitro. Eur Rev Med Pharmacol Sci 2011;15(2):129-34.

72. O'Flaherty C, de Lamirande E, Gagnon C. Reactive oxygen species and protein kinases modulate the level of phospho-MEK-like proteins during human sperm capacitation. Biol Reprod 2005;73(1):94-105.

73. Cohen-Bacrie $P$, Belloc $S$, Menezo YJR, Clement $P$, Hamidi J, Benkhalifa M. Correlation between DNA damage and sperm parameters: a prospective study of 1,633 patients. Fertil Steril 2009;91(5):1801-5. 